\title{
Criblage phytochimique et activité antimicrobienne de six rhizomes comestibles utilisés en médecine traditionnelle à Lubumbashi (RDC)
}

\author{
Valentin Chiribagula BASHIGE ${ }^{1,2,3^{*}}$, Amuri Salvius BAKARI ${ }^{1,2}$, \\ Philippe Ndjolo OKUSA ${ }^{2}$, Emery Mutombo KALONDA ${ }^{3}$ et Jean Baptiste Simbi LUMBU ${ }^{3}$
}

${ }^{1}$ Laboratoire de Pharmacognosie, Faculté des Sciences Pharmaceutiques Université de Lubumbashi, 27 av Kato Commune Kampemba, Lubumbashi/ RD Congo.

${ }^{2}$ Laboratoire de Chimie Thérapeutique et Analyse des substances médicamenteuses, Faculté des Sciences Pharmaceutiques Université de Lubumbashi, 27 av Kato Commune Kampemba, Lubumbashi/ RD Congo.

${ }^{3}$ Laboratoire de chimie Organique, Faculté des Sciences Université de Lubumbashi, 02 Av De la Maternité Commune de Lubumbashi /RD Congo.

*Auteur correspondant ; E-mail: bashige.chiribagula@unilu.ac.cd; bachival@gmail.com ;

$$
\text { Tel : +243991398521 }
$$

\section{RESUME}

Les affections microbiennes constituent des pathologies dominantes en RDC où pour y faire face la population recourt aux plantes. Cette étude a été conduite en vue d'évaluer l'activité antimicrobienne et identifier des groupes phytochimiques à potentiel antimicrobien de six rhizomes comestibles, utilisées en médecine traditionnelle à Lubumbashi. Le criblage phytochimique réalisé par des réactions classiques en solution a permis d'identifier dans tous les rhizomes, des flavonoïdes, des saponines, des tannins et dans 66,7\% d'entre eux, des quinones et des terpénoïdes. La méthode de dilution a permis d'évaluer l'activité antibactérienne des extraits aqueux (EAQ) et méthanoliques (EME) qui ont tous été actif avec une intensité allant de très forte $(\mathrm{CMI}=1,7$ $\mu \mathrm{g} / \mathrm{mL})$ à moyenne $(\mathrm{CMI}=50 \mu \mathrm{g} / \mathrm{mL})$. Eriosema verdikii $(\mathrm{EME}: \mathrm{CMI}=1,7 \mu \mathrm{g} / \mathrm{mL}$ sur $N$. meningitidis et $S$. aureus) a présenté la meilleure activité antibactérienne et Imperata cylindrica (EAQ : CMI = 1,7 $\mu \mathrm{g} / \mathrm{mL}$ sur Trichophyton rubrum), la meilleure activité antifongique. Le potentiel antimicrobien de ces rhizomes mis en évidence in vitro dans cette étude, fait des espèces étudiées des candidates à des investigations approfondies pouvant aboutir à la mise au point des médicaments traditionnels améliorés ou à la découverte de nouvelles molécules antibactériennes.

(C) 2020 International Formulae Group. All rights reserved.

Mots clés : antifongique, antibactérien, criblage chimique, rhizomes comestibles, Lubumbashi.

\section{Phytochemical Screening and Antimicrobial Activity of Six Edible Rhizomes Used in Traditional Medicine in Lubumbashi (DRC)}

\begin{abstract}
Microbial infections are dominant pathologies in the DRC where, to deal with them, several people resort to plants used in traditional medicine. This assesses the antimicrobial activity of six edible rhizomes used in traditional medicine in the city of Lubumbashi (DRC) and identifies phytochemical groups. Phytochemical
\end{abstract}


screening by solution reactions allowed the identification of flavonoids, saponins and tannins in all rhizomes, and in $66.7 \%$ of the rhizomes were found quinones and terpenoids. The dilution method allowed the evaluation of antibacterial activity, aqueous (EAQ) and methanolic (EME) extracts. This varies from very high (MIC $=1.7$ $\mu \mathrm{g} / \mathrm{mL}$ ) to medium (MIC $=50 \mu \mathrm{g} / \mathrm{mL}$ ). Eriosema verdikii (EME: $\mathrm{MIC}=1.7 \mu \mathrm{g} / \mathrm{mL}$ on $N$. meningitidis and S. aureus) has the greatest antibacterial in the collection and Imperata cylindrica (EAQ: MIC $=1.7 \mu \mathrm{g} / \mathrm{mL}$ On Trichophyton rubrum), the greatest antifungal activity. The results obtained by this study show that the six rhizomes studied, along with their food virtues, possess antibacterial and antifungal properties that can explain their use in traditional medicine against various conditions; In addition, the various phytochemical groups within them would play an important role in the proven virtues.

(C) 2020 International Formulae Group. All rights reserved.

Keywords: antifungal, antibacterial, chemical screening, edible rhizomes, Lubumbashi.

\section{INTRODUCTION}

Les affections microbiennes constituent une des catégories des pathologies les plus dominantes aussi bien dans les pays développés que dans les pays en voie de développement à l'image de la RDC (Madrid et al., 2017 ; Seale et al., 2017). Face à la recrudescence de ces maladies, l'industrie pharmaceutique a développé de molécules antimicrobiennes dont l'impact a vite été anéanti par le développement des phénomènes de résistance (Schwarz et al., 2017). La découverte de nouvelles substances efficaces sur les microorganismes résistants, constitue une grande urgence et justifie la conduite de plusieurs études en pharmacognosie et phytochimie. Dans les pays en voie de développement, où les infections bactériennes fauchent les plus des vies humaines, une fraction importante de la population recourt à la médecine traditionnelle (Nadimpalli, 2016). Celle-ci constitue une importante source de découverte de nouvelles substances bioactives (Hwang, 2019) comme le montrent plusieurs études ethnopharmacologiques ayant conduit à la découverte de substances antimicrobiennes (Raja et al., 2010 ; Tatsimo et al., 2017). Parmi ces nombreuses plantes médicinales investiguées figurent certaines plantes à rhizomes à la fois comestibles et antimicrobiennes. C'est le cas de Cyperus rotundus L. (Cyperaceae) (Zhang et al., 2017) utilisée en médecine traditionnelle Pakistanaise (Umair et al., 2017) ou de Polypodium vulgare L. (Polypodiaceae), utilisée en médecine traditionnelle Polonaise (Gleńsk et al., 2019).
Dans cette étude, les rhizomes comestibles de six plantes utilisées en médecine traditionnelle congolaise à Lubumbashi sont étudiés du point de vue de leur activité antimicrobienne sur Neisseria meningitidis, Salmonella typhi, Staphylococcus aureus, Streptococcus pneumoniae, Candida albicans et Trichophyton rubrum, mais également du point de vue phytochimique à la recherche de métabolites secondaires à potentiel antimicrobien. Ces rhizomes ont en commun la particularité d'être utilisées en médecine traditionnelle à Lubumbashi dans les affections microbiennes incluant l'abcès, la fièvre typhoïde, les gastroentérites, la pneumonie, la méningite, le pied d'athlète et les stomatites sans qu'aucune étude scientifique n'atteste de cette efficacité.

\section{MATERIEL ET METHODES}

\section{Matériel végétal}

Cyperus alternifolius L (Cyperaceae ; LMAG1110136 ; Lat. Sud 1148'12,1"Long. Est $27^{\circ} 27^{\prime 27,6 " ~ A l t . ~} 1258 \mathrm{~m}$ ), Echinochloa pyramidalis (Lam.) Hitche \& Chas (Poaceae; LMAG1110137; Lat. Sud 1148'32,5" Long. Est $27^{\circ} 27^{\prime} 17,9^{\prime \prime} \quad$ Alt. $1257 \quad \mathrm{~m}$ ), Eriosema verdickii De Wild (Leguminosae; LMAG1110138; Lat. Sud 11 ${ }^{\circ} 48^{\prime} 12,1^{\prime \prime}$ Long. EO $27^{\circ} 27^{\prime} 34,8^{\prime} \quad$ Alt. $1258 \mathrm{~m}$ ), Imperata cylindrica (L) Beauv. (Poaceae;

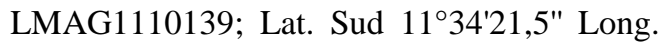
Est $27^{\circ} 22^{\prime}$ 57,4' Alt. $1267 \mathrm{~m}$ ), Typha angustifolia L. (Typhaceae; LMAG1110140; Lat. Sud 11 $48^{\prime}$ 06,9" Long. EO $27^{\circ} 27^{\prime} 36,3^{\prime \prime}$ Alt. $1291 \mathrm{~m}$ ) et Zingiber officinale Roscoe 
(Zingiberaceae; LMAG1110141; Lat. Sud $11^{\circ} 48^{\prime} 26,5^{\prime \prime}$ Long. Est $27^{\circ} 27^{\prime} 22,8^{\prime}$ Alt. $1291 \mathrm{~m}$ ) ont été récoltés en novembre 2017 en compagnie d'un ethnobotaniste, dans les alentours de la ferme Kikontwe à $15 \mathrm{~km}$ de la partie sud de la ville de Lubumbashi en République Démocratique du Congo (RDC), dans la galerie forestière Fipango. A cette occasion, des coordonnées GPSs ont été enregistrées et un herbier constitué à cet effet a été déposé à l'herbarium de la Faculté des Sciences Agronomiques de l'Université de Lubumbashi (RDC) où l'identité a été confirmée. Ces plantes ont été choisies du fait qu'elles sont localement utilisées à la fois comme aliments et plantes médicinales à Lubumbashi dans des affections communes sans que des preuves scientifiques n'attestent leurs usages médicinaux. Le matériel végétal récolté a été séché à l'ombre, à l'air libre, puis broyé (moulin en inox, Plymix, Belgique) et la poudre obtenue a servi à la préparation d'extraits.

\section{Souches microbiennes}

Le support microbien a été constitué de Neisseria meningitidis, Salmonella typhi, Staphylococcus aureus, Streptococcus pneumoniae, Candida albicans et Trichophyton rubrum. Ces isolats cliniques identifiés par galerie AI étaient issus des sujets congolais ayant présenté une résistance aux tétracyclines et à la nystatine. Ils sont responsables de la plupart d'affections pour lesquelles les plantes retenues sont utilisées à Lubumbashi et ont été fournis par le Laboratoire Provincial d'Analyses Biomédicales de Lubumbashi, structure de la Division Provinciale de la Santé dans la province du Haut-Katanga en RD Congo.

\section{Obtention d'extraits}

Les extraits aqueux ont été préparés en imitant la préparation des recettes utilisées en médecine traditionnelle, par décoction de 100 $\mathrm{g}$ des feuilles sèches broyées dans $1500 \mathrm{~mL}$ d'eau distillée. Les extraits méthanoliques ont été obtenus selon la procédure d'extraction utilisée par Bashige et al. (2018), par macération pendant 24 heures, de $250 \mathrm{~g}$ de poudre des drogues végétales dans $1000 \mathrm{~mL}$ de méthanol (34860-2.5L-R, Sigma-Aldrich). Tous les extraits ont été concentrés sous pression réduite (130-180 mbar) à l'aide d'un évaporateur rotatif (Rotavapor Büchi R-100 29/32 Version V - 230V), entre 36 et $40{ }^{\circ} \mathrm{C}$.

\section{Préparation de l'inoculum standard}

Les suspensions de l'inoculum de bactéries ou de fungi ont été préparées selon la technique décrite par WHO (2003), en dispersant des souches pures dans du Bouillon thioglycolate avec résazurine (42 074, bioMérieux) et cultivé à $37{ }^{\circ} \mathrm{C}$ pendant $24 \mathrm{~h}$ (Bactérie) ou $28{ }^{\circ} \mathrm{C}$ (fungi). La turbidité de la suspension microbienne a été ajustée avec un densitomètre (DEN-1, BS-050102-AAF, Biosan) à un standard de $0,5 \mathrm{Mc}$ Farland équivalent à environ 1 à $5 \times 10^{8}$ cellules microbiennes comptées $/ \mathrm{mL}$. Cette suspension a été diluée au centième, constituant ainsi l'inoculum standard (Bashige et al., 2018).

\section{Référence au témoin}

Quatre témoins ont été utilisés. Le premier témoin a été constitué uniquement du milieu de culture $(2 \mathrm{~mL})$ pour vérifier la stérilité du milieu de travail. Le deuxième contenait le milieu de culture et l'extrait $(1 \div 1)$ pour vérifier la stérilité des extraits et disposer de la référence du système extrait-milieu de culture. Le troisième a été constitué de $0,1 \mathrm{~mL}$ de DMSO (276855-100ML, Sigma-Aldrich) et $1,9 \mathrm{~mL}$ de l'inoculum pour vérifier la viabilité des germes vis-à-vis du DMSO et le quatrième, du milieu de culture et de l'érythromycine (E5389-1G, Sigma-Aldrich) comme substance antibactérienne de référence, pour apprécier le comportement d'une substance active dans le milieu.

\section{Détermination de la concentration minimale inhibitrice (CMI)}

L'évaluation de l'activité antimicrobienne a été réalisée par la méthode de dilution. Elle consiste à mettre en interaction les germes et les extraits, à différentes dilutions, et à observer l'activité par l'absence visuelle de croissance des microorganismes après un temps d'incubation (Balouiri et al., 
2016). Les solutions mères d'extraits ont été préparées en dissolvant $1 \mathrm{mg}$ d'extrait sec dans $100 \mu \mathrm{L}$ de DMSO puis additionnées de 1900 $\mu \mathrm{L}$ du milieu de culture. Cinq dilutions d'ordre 2 ont été réalisées (de $50 \mu \mathrm{g} / \mathrm{mL}$ à $1,56 \mu \mathrm{g} / \mathrm{mL}$ ) pour chaque extrait et placées dans différents tubes aseptiques. En suite, $1000 \mu \mathrm{L}$ de l'inoculum standard ont été ajoutés dans chaque tube et le mélange a été incubé pendant $24 \mathrm{~h}$ à $37^{\circ} \mathrm{C}$ (bactéries) et à $28^{\circ} \mathrm{C}$ (fungi), puis la croissance des microorganismes a été observée visuellement. La concentration minimale inhibitrice a été définie comme la plus faible concentration à laquelle l'extrait a empêché la croissance visible des bactéries (Bashige-Chiribagula et al., 2017a).

\section{Détermination de la concentration minimale bactéricide (CMB) ou fongicide (CMF)}

Le prélèvement s'est effectué dans les tubes ayant servi à la détermination des CMI. L'ensemencement s'est effectué dans les boites de Pétri (067.000000.65, Pyrex D.120MM H.20MM PACK-10, Humeau) sur milieu Gélose salmonella-shigella (145.007667.54, 7667-500G, Merck) pour les bactéries et gélose - sabouraud (550.610103.54, 610103-500G, LIOFILCHEM) pour les champignons et l'incubation s'est réalisée à $37{ }^{\circ} \mathrm{C}$ pendant $24 \mathrm{~h}$ (Bactérie) et $28{ }^{\circ} \mathrm{C}$ (champignons) avant la visualisation de la croissance microbienne. En accord avec Hoşgor et al. (2011) ainsi que Kaya et al. (2012), la CMB a été définie comme la plus petite concentration à laquelle l'extrait a empêché la croissance visible des bactéries après repiquage et la CMF, la plus petite concentration à laquelle l'extrait a empêché la croissance visible des champignons après repiquage.

\section{Effet des extraits et leur catégorisation}

L'effet des extraits a été déterminé en effectuant le rapport CMB (ou CMF) /CMI. Lorsque ce rapport est égal à 1, l'antibiotique est dit «bactéricide absolu» (Plummer et al., 2004) ou « fongicide absolu », s'il est inférieur ou égal à 4, l'antibiotique est dit «bactéricide» (ou «fongicide»), s'il est supérieur à 4 , l'antibiotique est dit simplement «bactériostatique » (ou «fongistatique $)$
(Carbonnelle et al., 1987 ; Gatsing et al., 2006). Les extraits ont été catégorisés en 4 classes : i) extraits à très forte activité : si $\mathrm{CMI} \leq 5$ $\mu \mathrm{g} / \mathrm{mL}$; ii) extrait à forte activité si $5 \mu \mathrm{g} / \mathrm{mL} \leq$ CMI $\leq 50 \mu \mathrm{g} / \mathrm{mL}$; iii) extrait à activité modérée si $50 \mu \mathrm{g} / \mathrm{mL} \leq \mathrm{CMI} \leq 325 \mu \mathrm{g} / \mathrm{mL}$ et iv) extrait à faible activité si CMI $>325 \mu \mathrm{g} / \mathrm{mL}$ (Kuete, 2010).

\section{Criblage chimique par des réactions en solution}

Les réactions classiques en solution basées sur l'apparition de la coloration pour les anthocyanes, les flavonoïdes, les quinones, les stéroïdes, les tannins et les terpénoïdes, la précipitation pour les alcaloïdes ou la formation de mousse pour les saponines, ont permis de réaliser des tests phytochimiques, qui ont consisté à rechercher de groupes des substances bioactives précités, parmi lesquels certains seraient pourvus d'un potentiel antimicrobien. La recherche des hétérosides cyanogènes, avait pour objectif d'identifier une éventuelle toxicité due au cyanure.

Les alcaloïdes ont été mis en évidence en les précipitant à l'aide de six réactifs à savoir : Bertrand, Dragendorf, Hager, Mayer, Sonneschein (Bashige et al., 2018). Le résultat n'a été considéré comme positif que lorsque tous ces réactifs donnaient un précipité avec l'extrait. Les flavonoïdes ont été identifiés avec le réactif de Shinoda lorsque le mélange extrait aqueux acide et copeaux de magnésium (AE61.2, Roth) faisait apparaitre une coloration rose-rouge ou rouge-violacée ; Quant aux anthocyanes, leur coloration rouge en l'absence des copeaux de magnésium dans le mélange précédent, les identifiait (BashigeChiribagula et al., 2017b). Les hétérosides cyanogènes ont été mis en évidence par la réaction de l'acide picrique avec la vapeur d'une décoction aqueuse lorsque le papier picrosodé (Acide picrique 1\%: 197378-500G, Sigma-Aldrich) virait à l'orange ou au rouge (Bashige et al., 2018). Les quinones ont été recherchées en faisant réagir les extraits éthérés avec le $\mathrm{KOH} \mathrm{1 \%} \mathrm{(P031.3,} \mathrm{Roth).} \mathrm{L'apparition}$ d'une coloration caractéristique allant de rouge-orangé au violet-pourpre indiquait leur présence. Les saponines ont été recherchés par 
leur capacité à former une mousse (hauteur : > $10 \mathrm{~mm}$ ) après agitation d'une solution aqueuse les contentant (Longanga et al., 2000). Les stéroïdes ont été recherchés par la réaction de Lieberman-Burchard. Elle a consisté à mettre l'extrait organique éthéré (296082-2.5L, Sigma-Aldrich) en présence de l'acide acétique anhydre (242845, Sigma-Aldrich) et de l'acide sulfurique concentré (339741-500ML, SigmaAldrich) et observer une coloration mauve ou verte. Les tannins ont été recherchés en faisant réagir un infusé aqueux avec une solution de chlorure ferrique à 1\% (157740-100G, SigmaAldrich). La réaction n'a été considérée comme positive qu'avec la formation des précipités bleu-vert, bleu sombre ou vert (BashigeChiribagula et al., 2017b). Le mélange de l'infusé avec le réactif de Stiasny a identifié les tannins catéchiques alors que l'ajout au filtrat de l'acétate de sodium et du chlorure ferrique entrainant la formation des précipités, a identifié les tannins galliques (Mustapha et al., 2016).
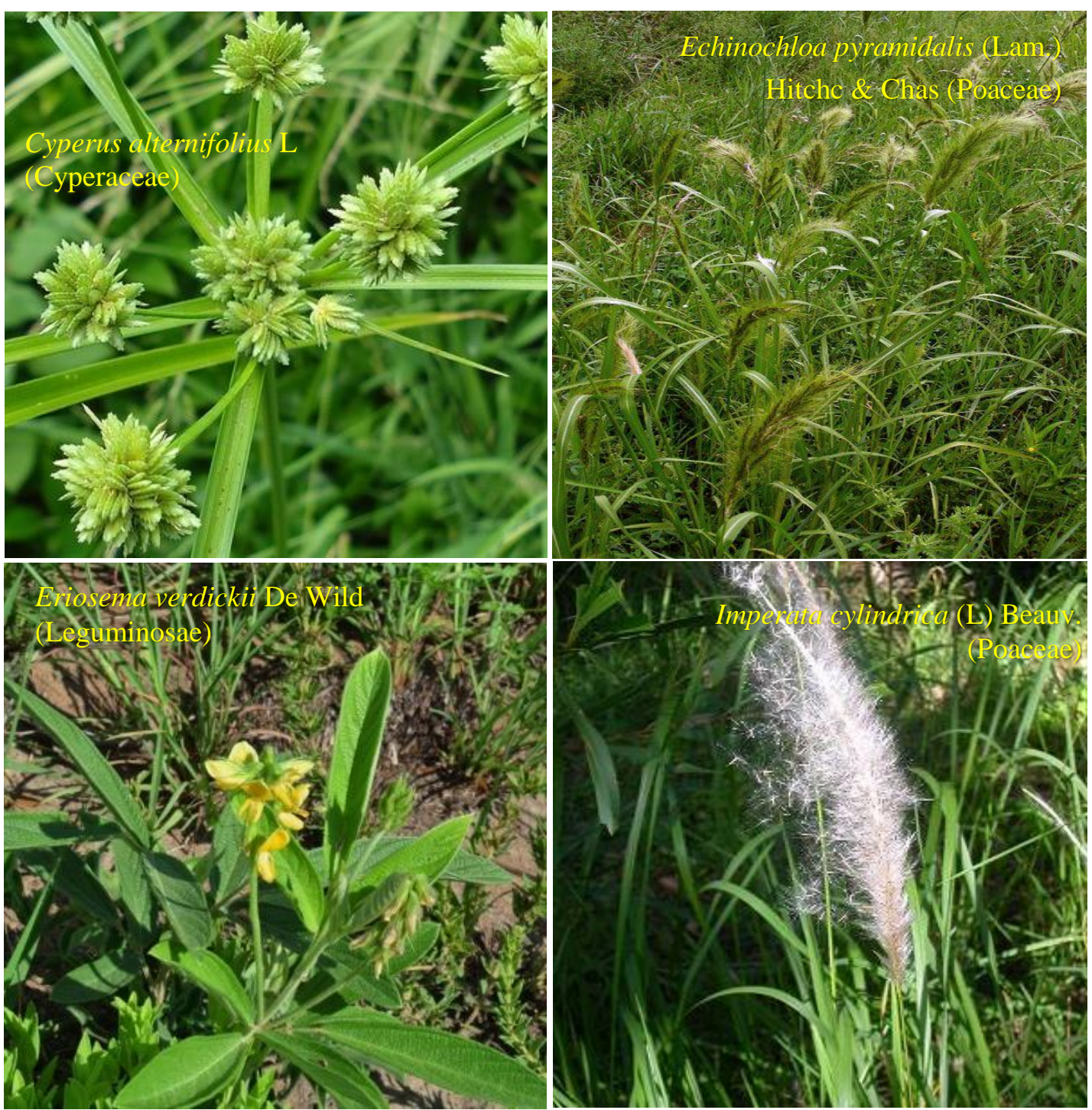

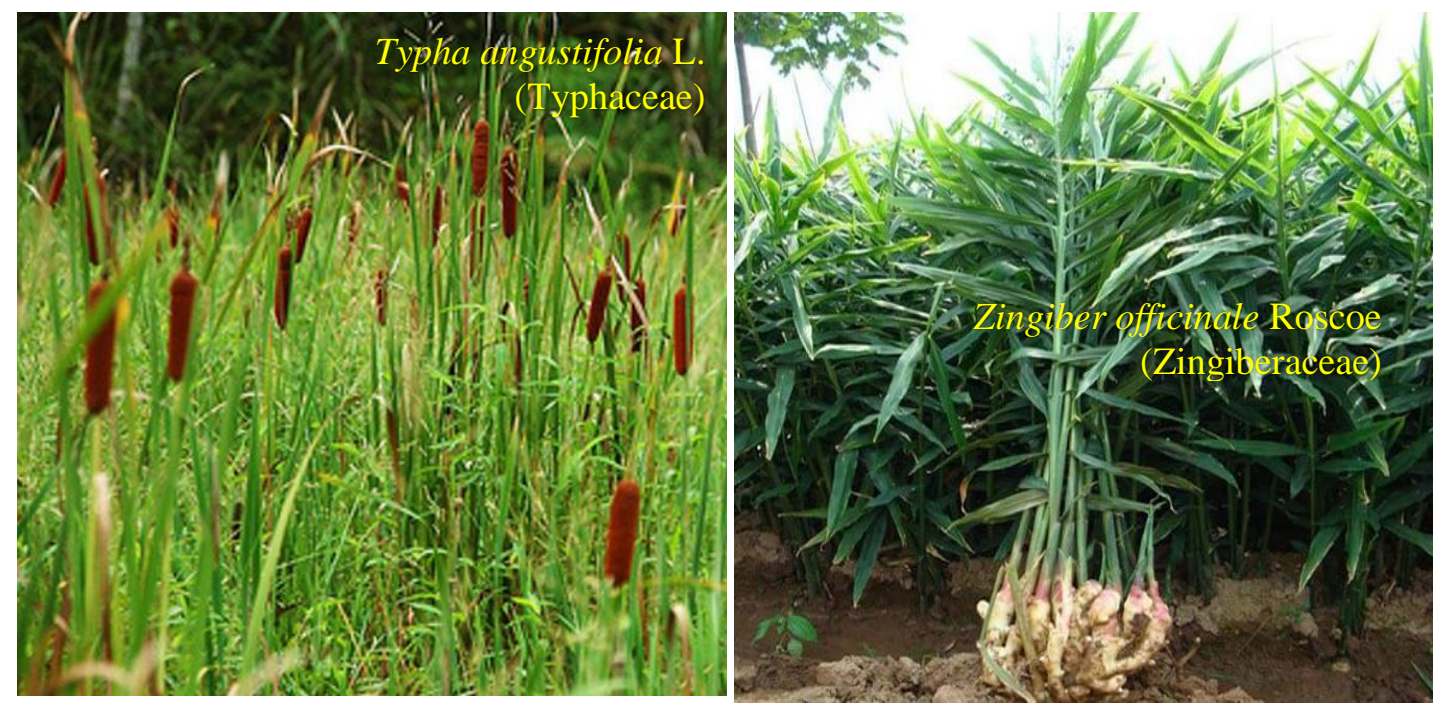

Figure 1: Plantes à rhizomes comestibles et utilisés en médecine traditionnelle lushoise (RDC).

\section{RESULTATS}

\section{Activité antibactérienne des extraits}

Quarante-huit tests (12 tests par germe) ont été effectués pour l'évaluation de l'activité antibactérienne. Les extraits présentent une activité allant de très forte $(\mathrm{CMI}=1,7 \mu \mathrm{g} / \mathrm{mL})$ à moyenne $(\mathrm{CMI}=50 \mu \mathrm{g} / \mathrm{mL})$. Cette activité ne suit pas une tendance univoque : tantôt les extraits aqueux (EAQ) sont plus actifs que les extraits méthanoliques, comme sur Streptococcus pneumoniae et sur Neisseria meningitidis, tantôt c'est l'inverse, comme sur Streptococcus aureus, tantôt l'activité est la même, le cas observé sur C. albicans. Eriosema verdikii (EME : CMI $=1,7 \mu \mathrm{g} / \mathrm{mL}$ sur $N$. meningitidis et sur $S$. aureus) dispose de la meilleure activité antibactérienne de la collection et Imperata cylindrica (EAQ : CMI $=1,7 \mu \mathrm{g} / \mathrm{mL}$ sur Trichophyton rubrum), la meilleure activité antifongique.

\section{Activité antibactérienne sur Neisseria meningitidis}

L'activité antibactérienne sur Neisseria meningitidis totalement bactéricide absolue, a été observée aux concentrations variantes entre 3,1 et $50 \mu \mathrm{g} / \mathrm{mL}$ (Tableau 1). L'extrait méthanolique de Eriosema verdikii a présenté la meilleure activité sur cette souche. Par ailleurs, l'activité des extraits méthanoliques a été deux fois supérieure à celle des extraits aqueux (Tableau 1).

\section{Activité antibactérienne sur Salmonella typhi}

L'activité antibactérienne sur Salmonella typhi est passée de très forte (CMI $=1,7 \mu \mathrm{g} / \mathrm{mL}$ chez I. cylindrica pour EME) à moyenne $(\mathrm{CMI}=25 \mu \mathrm{g} / \mathrm{mL}$ pour EA) et tous les extraits ont présenté un effet bactéricide. Dans environ $70 \%$ des cas, l'activité antibactérienne des extraits méthanoliques est supérieure à celle des extraits aqueux (Tableau 2).

\section{Activité antibactérienne sur Streptococcus pneumoniae}

Sur Streptococcus pneumoniae, les extraits ont présenté une activité allant de très forte (Echinochloa pyramidalis : $\mathrm{CMI}=1,7$ $\mu \mathrm{g} / \mathrm{mL})$ à moyenne $(\mathrm{CMI}=25 \mu \mathrm{g} / \mathrm{mL})$. Tous les extraits ont présenté un effet bactéricide absolu. Les extraits aqueux sont plus actifs que les extraits méthanoliques (Tableau 3).

\section{Activité antibactérienne sur Staphylococcus aureus}

L'activité des différents extraits sur Staphylococcus aureus a variée de très forte (Eriosema verdikii, CMI $=1,7 \mu \mathrm{g} / \mathrm{mL}$ pour EME) à moyenne $(\mathrm{CMI}=12,5 \mu \mathrm{g} / \mathrm{mL})$. L'activité de l'extrait aqueux est deux fois inférieure à celle de l'extrait méthanolique et plus de $66 \%$ d'extraits présentent un effet bactéricide absolu (Tableau 4). 


\section{Activité antifongique des extraits}

Pour l'ensemble des tests effectués sur les souches fongiques (24 tests), l'activité a varié de très forte (EME de Eriosema verdikii, $\mathrm{CMI}=1,7 \mu \mathrm{g} / \mathrm{mL})$ à moyenne (EAQ, CMI = $50 \mu \mathrm{g} / \mathrm{mL}$. Comparativement à Trichophyton rubrum, Candida albicans présente une plus grande sensibilité aux extraits.

\section{Activité antifongique sur Candida albicans}

Sur $C$. albicans, l'activité antifongique de différents extraits est la même (CMI $=3,1$ $\mu \mathrm{g} / \mathrm{mL}$ ) et toutes les plantes présentent un effet fongicide absolu à l'exception de Typha angustifolia et Zingiber officinale qui présentent un effet fongicide (Tableau 5).

\section{Activité antifongique sur Trichophyton rubrum}

L'activité antifongique sur $T$. rubrum varie entre très active $(\mathrm{CMI}=1,7 \mu \mathrm{g} / \mathrm{mL}) \mathrm{EAQ}$ de Imperata cylindica et moyenne $(\mathrm{CMI}=50$ $\mu \mathrm{g} / \mathrm{mL}$ ) avec tous les extraits de $C$. alternifolius et $E$. pyramidalis. Cette activité est essentiellement fongicide et dans le cas contraire non déterminée (Tableau 6).

\section{Criblage phytochimique}

Le criblage phytochimique a révélé la présence d'au moins 5 groupes à potentiel antimicrobien sur les huit recherchés. Tous les rhizomes contiennent des flavonoïdes, des saponines, des tannins. Imperata cylindrica est l'espèce la plus riche et contient les trois quarts des groupes recherchés (Tableau 7).

Les recettes utilisées en médecine traditionnelle à base de ces rhizomes se fond par décoction, ce qui peut faire craindre une intoxication au cyanure présente sous forme d'hétérosides libérable in situ. Les résultats obtenus dans cette étude montrent que ce mode de préparation utilisée en médecine traditionnelle n'expose pas à une éventuelle intoxication due au cyanure, du fait qu'une réaction négative a été observée dans tous les extraits lors de la recherche des hétérosides cyanogènes (Tableau 7).

Tableau 1 : Activité antibactérienne des extraits des rhizomes de six plantes sélectionnées sur Neisseria meningitidis.

\begin{tabular}{lrrrrrrl}
\hline & \multicolumn{2}{c}{ CMI $(\boldsymbol{\mu g} / \mathbf{m L})$} & \multicolumn{2}{l}{ CMB $(\boldsymbol{\mu g} / \mathbf{m L})$} & \multicolumn{2}{l}{ CMB/CMI } & \multicolumn{2}{l}{ Effet } \\
\cline { 2 - 8 } Espèce végétale & EAQ & EME & EAQ & EME & EAQ & EME & EEA, EEM \\
\hline Cyperus alternifolius & 6,3 & 3,1 & 6,3 & 3,1 & 1 & 1 & BA, BA \\
Echinochloa pyramidalis & 6,3 & 3,1 & 6,3 & 3,1 & 1 & 1 & BA, BA \\
Eriosema verdikii & 3,1 & 1,7 & 6,3 & 1,7 & 1 & 1 & BA, BA \\
Imperata cylindrica & 12,5 & 6,3 & 12,5 & 6,3 & 1 & 1 & BA, BA \\
Typha angustifolia & 50 & 12,5 & 50 & 12,5 & 1 & 1 & BA, BA \\
Zingiber officinale & 50 & 12,5 & 50 & 12,5 & 1 & 1 & BA, BA
\end{tabular}

EAQ : extrait aqueux, EME : extrait méthanolique ; EDCM : extrait dichlorométhanique ; BC : Bactéricide ; BA : Bactéricide absolu, ND : Non déterminé, EEA : effet de l'extrait aqueux, EEM : Effet de l'extrait méthanolique. 
Tableau 2 : Activité antibactérienne des extraits des rhizomes de six plantes sélectionnées sur Salmonella typhi.

\begin{tabular}{lrrrrrrl}
\hline & \multicolumn{2}{c}{ CMI $(\boldsymbol{\mu g} / \mathbf{m L})$} & \multicolumn{2}{l}{ CMB $(\boldsymbol{\mu g} / \mathbf{m L})$} & \multicolumn{2}{l}{ CMB/CMI } & \multicolumn{2}{l}{ Effet } \\
\cline { 2 - 8 } Espèce végétale & EAQ & EME & EAQ & EME & EAQ & EME & EEA, EEM \\
\hline Cyperus alternifolius & 12,5 & 3,1 & 25 & 6,3 & 2 & 2 & BC, BC \\
Echinochloa pyramidalis & 12,5 & 3,1 & 25 & 6,3 & 2 & 2 & BC, BC \\
Eriosema verdikii & 3,1 & 3,1 & 6,3 & 6,3 & 2 & 2 & BC, BC \\
Imperata cylindrica & 3,1 & 1,7 & 6,3 & 3,1 & 2 & 2 & BC, BC \\
Typha angustifolia & 6,3 & 3,1 & 12,5 & 6,3 & 2 & 2 & BC, BC \\
Zingiber officinale & 3,1 & 3,1 & 6,3 & 6,3 & 2 & 2 & BC, BC
\end{tabular}

EAQ : extrait aqueux, EME : extrait méthanolique ; EDCM : extrait dichlorométhanique ; BC: Bactéricide; BA : Bactéricide absolu, ND : Non déterminé, EEA : effet de l'extrait aqueux, EEM : Effet de l'extrait méthanolique.

Tableau 3: Activité antibactérienne des extraits des rhizomes de six plantes sélectionnées sur Streptococcus pneumoniae.

\begin{tabular}{|c|c|c|c|c|c|c|c|}
\hline \multirow[b]{2}{*}{ Espèce végétale } & \multicolumn{2}{|c|}{ CMI $(\mu \mathrm{g} / \mathrm{mL})$} & \multicolumn{2}{|c|}{ CMB $(\mu \mathrm{g} / \mathrm{mL})$} & \multicolumn{2}{|c|}{ CMB/CMI } & \multirow{2}{*}{$\begin{array}{l}\text { Effet } \\
\text { EEA, EEM }\end{array}$} \\
\hline & EAQ & EME & EAQ & EME & EAQ & EME & \\
\hline Cyperus alternifolius & 12,5 & 25 & 12,5 & 25 & 1 & 1 & $\mathrm{BA}, \mathrm{BA}$ \\
\hline Echinochloa pyramidalis & 1,7 & 3,1 & 1,7 & 3,1 & 1 & 1 & $\mathrm{BA}, \mathrm{BA}$ \\
\hline Eriosema verdikii & 3,1 & 6,1 & 3,1 & 6,1 & 1 & 1 & $\mathrm{BA}, \mathrm{BA}$ \\
\hline Imperata cylindrica & 6,1 & 12,5 & 6,1 & 12,5 & 1 & 1 & $\mathrm{BA}, \mathrm{BA}$ \\
\hline Typha angustifolia & 12,5 & 25 & 12,5 & 25 & 1 & 1 & $\mathrm{BA}, \mathrm{BA}$ \\
\hline Zingiber officinale & 12,5 & 25 & 12,5 & 25 & 1 & 1 & $\mathrm{BA}, \mathrm{BA}$ \\
\hline
\end{tabular}

EAQ : extrait aqueux, EME : extrait méthanolique ; EDCM : extrait dichlorométhanique ; BC : Bactéricide ; BA : Bactéricide absolu, ND : Non déterminé, EEA : effet de l'extrait aqueux, EEM : Effet de l'extrait méthanolique.

Tableau 4: Activité antibactérienne des extraits des rhizomes de six plantes sélectionnées sur Staphylococcus aureus.

\begin{tabular}{lrrrrrrl}
\hline & \multicolumn{2}{c}{ CMI $(\boldsymbol{\mu g} / \mathbf{m L})$} & \multicolumn{2}{l}{ CMB $(\boldsymbol{\mu g} / \mathbf{m L})$} & \multicolumn{2}{l}{ CMB/CMI } & \multicolumn{2}{l}{ Effet } \\
\cline { 2 - 9 } Espèce végétale & EAQ & EME & EAQ & EME & EAQ & EME & EEA, EEM \\
\hline Cyperus alternifolius & 6,3 & 3,1 & 6,3 & 3,1 & 1 & 1 & BA, BA \\
Echinochloa pyramidalis & 6,3 & 3,1 & 6,3 & 3,1 & 1 & 1 & BA, BA \\
Eriosema verdikii & 3,1 & 1,7 & 3,1 & 3,1 & 1 & 1 & BA, BA \\
Imperata cylindrica & 12,5 & 6,3 & 12,5 & 12,5 & 1 & 1 & BA, BA \\
Typha angustifolia & 6,3 & 3,1 & 12,5 & 6,3 & 2 & 2 & BC, BC \\
Zingiber officinale & 12,5 & 3,1 & 25 & 6,3 & 2 & 2 & BC, BC \\
\hline
\end{tabular}


EAQ : extrait aqueux, EME : extrait méthanolique ; EDCM : extrait dichlorométhanique ; BC : Bactéricide ; BA : Bactéricide absolu, ND : Non déterminé, EEA : effet de l'extrait aqueux, EEM : Effet de l'extrait méthanolique.

Tableau 5 : Activité antibactérienne des extraits des rhizomes de six plantes sélectionnées sur Candida albicans.

\begin{tabular}{lrrrrrrl}
\hline \multirow{2}{*}{ Espèce végétale } & \multicolumn{2}{c}{ CMI $(\boldsymbol{\mu g} / \mathbf{m L})$} & \multicolumn{2}{l}{ CMF $(\boldsymbol{\mu g} / \mathbf{m L})$} & \multicolumn{2}{l}{ CMF/CMI } & \multicolumn{2}{l}{ Effet } \\
\cline { 2 - 8 } & EAQ & EME & EAQ & EME & EAQ & EME & EEA, EEM \\
\hline Cyperus alternifolius & 3,1 & 3,1 & 3,1 & 3,1 & 1 & 1 & FA, FA \\
Echinochloa pyramidalis & 3,1 & 3,1 & 3,1 & 3,1 & 1 & 1 & FA, FA \\
Eriosema verdikii & 3,1 & 3,1 & 3,1 & 3,1 & 1 & 1 & FA, FA \\
Imperata cylindrica & 3,1 & 3,1 & 3,1 & 3,1 & 1 & 1 & FA, FA \\
Typha angustifolia & 3,1 & 3,1 & 6,3 & 6,3 & 2 & 2 & FC, FC \\
Zingiber officinale & 3,1 & 3,1 & 6,3 & 6,3 & 2 & 2 & FC, FC \\
\hline
\end{tabular}

EAQ : extrait aqueux, EME : extrait méthanolique ; EDCM : extrait dichlorométhanique ; FC : Fongicide ; FA : Fongicide absolu, ND : Non déterminé, EEA : Effet de l'extrait aqueux, EEM : Effet de l'extrait méthanolique.

Tableau 6: Activité antibactérienne des extraits des rhizomes de six plantes sélectionnées sur Trichophyton rubrum.

\begin{tabular}{lrrrrrrrr}
\hline \multirow{2}{*}{ Espèce végétale } & \multicolumn{3}{c}{ CMI $(\boldsymbol{\mu g} / \mathbf{m L})$} & \multicolumn{2}{c}{ CMF $(\boldsymbol{\mu g} / \mathbf{m L})$} & \multicolumn{2}{c}{ CMF/CMI } & Effet \\
\cline { 2 - 8 } & \multicolumn{1}{c}{ EAQ } & EME & EAQ & EME & EAQ & EME & EEA, EEM \\
\hline Cyperus alternifolius & 50 & 50 & ND & ND & ND & ND & ND, ND \\
Echinochloa pyramidalis & 50 & 50 & ND & ND & ND & ND & ND, ND \\
Eriosema verdikii & 12,5 & 25 & 25 & 50 & 2 & ND & FC, ND \\
Imperata cylindrica & 1,7 & 3,1 & 3,1 & 6,3 & 2 & 2 & FC, FC \\
Typha angustifolia & 6,3 & 12,5 & 12,5 & 25 & 2 & 2 & FC, FC \\
Zingiber officinale & 3,1 & 6,3 & 6,3 & 12,5 & 2 & 2 & FC, FC \\
\hline
\end{tabular}

$E A Q$ : extrait aqueux, EME : extrait méthanolique ; EDCM : extrait dichlorométhanique ; FC : Fongicide ; FA : Fongicide absolu, $N D:$ Non déterminé, EEA : effet de l'extrait aqueux, EEM : Effet de l'extrait méthanolique.

Tableau 7: Résultats du criblage phytochimique de six rhizomes comestibles utilisées en médecine traditionnelle à Lubumbashi.

\begin{tabular}{lrrrrrrr}
\hline & CyAl & EcPy & ErVe & ImCy & TyAn & ZiOf & Fi (\%) \\
\hline Alcaloïdes & + & - & - & + & - & + & 50 \\
Anthocyanes & - & - & - & - & & + & 16,7 \\
Flavonoïdes & + & + & + & + & + & + & 100 \\
Quinones & - & + & + & - & + & + & 66,7 \\
Stéroïdes & + & + & + & + & - & + & 83,3 \\
Saponines & + & + & + & + & + & + & 100 \\
Tannins & + & + & + & + & + & + & 100 \\
Terpénoïdes & + & - & - & + & + & + & 66,7 \\
\hline
\end{tabular}




\begin{tabular}{lcccccc}
\hline Bilan (\%) & $\mathbf{7 5}$ & $\mathbf{6 2 , 5}$ & $\mathbf{6 2 , 5}$ & $\mathbf{7 5}$ & $\mathbf{6 2 , 5}$ & $\mathbf{1 0 0}$ \\
\hline $\mathrm{HCN}$ & - & - & - & - & - & - \\
\hline $\mathrm{Cy} \mathrm{Al}:$ Cyperus alternifolius, EcPy : Echinochloa pyramidalis, ErVe $:$ Eriosema verdikii, ImCy : Imperata cylindrica, TyAn : Typha \\
angustifolia, ZiOf : Zingiber officinale, Fi : fréquence de présence avec
\end{tabular}

\section{DISCUSSION}

Cette étude a évalué l'activité antimicrobienne, sur Neisseria meningitidis, Salmonella typhi, Staphylococcus aureus, Streptococcus pneumoniae, Candida albicans et Trichophyton rubrum, de six rhizomes comestibles, Cyperus alternifolius, Echinochloa pyramidalis, Eriosema verdikii, Imperata cylindrica, Typha angustifolia et Zingiber officinalis, utilisées en médecine traditionnelle Lushoise. Un criblage phytochimique a également été réalisé en vue d'en rechercher des métabolites secondaires à potentiel antimicrobien.

Il a été rapporté que les parties aériennes de Cyperus alternifolius sont douées des propriétés hépatoprotectrices et contiennent des terpénoïdes et des polyphénols notamment des flavonoïdes (Awaad et al., 2012). Les résultats obtenus dans cette étude (Tableau 7) montrent que ces métabolites sont également présents dans les rhizomes. Ces groupes phytochimiques seraient probablement responsables des activités antimicrobiennes des extraits méthanoliques observées sur $N$. meningitidis, S. typhi, S. aureus et C. albicans $(\mathrm{CMI}=3,1 \mu \mathrm{g} / \mathrm{mL})$ au cours de cette étude, comme rapporté dans les travaux antérieurs sur d'autres espèces (Sima-Obiang et al., 2018 ; William et al., 2019).

Cette étude rapporte la présence, des saponines et des stéroïdes et une activité antimicrobienne des rhizomes de Echinochloa pyramidalis avec une CMI de $3,1 \mu \mathrm{g} / \mathrm{mL}$ sur $C$. albicans, S. aureus, S. pneumoniae, S. typhi et $N$. meningitidis. Une activité antimicrobienne de E. stagmina, une plante du même genre, avait été rapprochée à la présence des stéroïdes et terpénoïdes, groupes à potentiel antimicrobien (Attanayaka et al., 2008; Adeshina et al., 2009), laissant ainsi suggérer que ces groupes phytochimiques seraient également responsables de l'activité observée avec E. pyramidalis.

La littérature ne livre aucune information phytochimique ni aucune activité biologique chez Eriosema verdikii. Les résultats obtenus dans la présente étude constituent donc une première information sur la présence de flavonoïdes, de quinones, de stéroïdes, de saponines et de tannins dans les rhizomes de cette plante, ainsi que sa forte activité antimicrobienne sur $C$. albicans, $S$. aureus, $S$. pneumoniae, $S$. typhi et $N$. meningitidis (CMI $\leq 3,1 \mu \mathrm{g} / \mathrm{mL})$; d'autres espèces du même genre, contenant aussi de flavonoïdes et de tannins ont montré une activité antimicrobienne sur $S$. aureus et $C$. albicans. Ce sont notamment E. robustum (Awouafack et al., 2013) et E. psoraleoides (Elechi et Igboh, 2017). Les flavonoïdes et les tannins rencontrés dans les espèces du genre Eriosema, pourraient être responsables de leurs activités thérapeutiques, principalement antibactérienne.

L'identification des flavonoïdes et stéroïdes dans Imperata cylindrica est en accord avec les travaux de Lalthanpuii et al. (2019). Au-delà de l'activité des extraits aqueux rapportée par Lalthanpuii et al. (2018) sur $E$. coli et $S$. aureus et confirmés ici, leur spectre de l'activité antimicrobienne est élargi par cette étude sur S. typhi, C. albicans et $T$. rubrum à la CMI de $3,1 \mu \mathrm{g} / \mathrm{mL}$.

Plusieurs groupes phytochimiques, notamment les flavonoïdes, les quinones, les saponines, les stéroïdes, les tannins et les terpénoïdes, identifiées dans le rhizome de Typha angustifolia au cours de cette étude sont rapportés par Amari et al. (2014). Aux activités antimicrobiennes sur $S$. typhimurum, $P$. aeroginosa et $E$. coli de ses parties aériennes comme le rapportent Varghese et al. (2009), la présente étude adjoint, à la lumière des 
résultats, une activité sur $C$. albicans, $S$. aureus et $S$. typhi des rhizomes. Les travaux de Varghese et al. (2009) ont attribué l'activité des parties aériennes sur $S$. aureus à deux flavonoïdes. L'identification des flavonoïdes dans les parties souterraines de la même espèce, douée en plus de la même activité antibactérienne, laisse penser que ces propriétés viendraient des flavonoïdes.

Zingiber officinalis a déjà fait l'objet de plusieurs investigations portant sur les aspects phytochimiques et antimicrobien de ses rhizomes (Haniadka et al., 2013 ; Gupta et al., 2014 ; Ashraf et al., 2017). Les résultats cette étude tant du point de vue phytochimique que microbiologique, confirment que la variété cultivée et consommée à Lubumbashi (RDC), a la même composition chimique (alcaloïdes, anthocyanes, flavonoïdes, quinones, saponines, stéroïdes, tannins, terpénoïdes) que les variétés d'ailleurs et est douée de propriétés antimicrobiennes similaires sur $S$. typhi, $S$. aureus, C. albicans et Trichophyton rubrum.

\section{Conclusion}

Cette étude a permis de poser les bases scientifiques de l'utilisation en medecine traditionnelle à Lubumbashi (RD Congo), de six rhizomes comestibles contre certaines affections bactériennes. Elle a mis en évidence pour la première fois, la composition chimique et l'activité antibactérienne du rhizome de Eriosema verdikii tout en élargissant la connaissance de 5 autres rhizomes, tous, à la fois comestibles et utilisées en médecine traditionnelle. Leur activité antibactérienne conforte l'approche des praticiens de la medecine traditionnelle à Lubumbashi et impose de recherches plus approfondies visant à déterminer les composés responsables de l'activité observée.

\section{CONFLITS D'INTERETS}

Les auteurs ne déclarent aucun conflit d'intérêts.

\section{CONTRIBUTIONS DES AUTEURS}

Conception du sujet, récolte et compilation des données, rédaction de la dissertation : VCB ; correction du texte : ASB, PNO, EMK; supervision de la recherche, encadrement et correction du style : JBSL.

\section{RÉFÉRENCES}

Adeshina GO, Ebere OCL, Onwuegbuchulam NO, Ehinmidu JO. 2009. Preliminary studies on antimicrobial activities of ethanolic extracts of Ficus sycomorus Linn. and Ficus platyphylla Del. (Moraceae). Int. J. Biol. Chem. Sci., 3(5): 1013-1020.

DOI: 10.4314/ijbcs.v3i5.51080

Amari NO, Bouzouina M, Berkani A, Lotmani B. 2014. Phytochemical screening and antioxidant capacity of the aerial parts of Thymelaea hirsuta L. Asian Pacific Journal of Tropical Disease, 4(2): 104109.

DOI: $10.1016 / \mathrm{S} 2222-$ 1808(14)60324-8.

Ashraf K, Sultan S, Ali Sha SA. 2017. Phychemistry, Phytochemical, Pharmacological and Molecular Study of Zingiber Officinale Roscoe: A Review. Int J Pharm Pharm Sci, 9(11): 8-16. DOI: https://doi.org/10.22159/ijpps.2017 v9i11.19613.

Attanayaka K, Mendis S, Jansz E, Ekanayake S, Perera A. 2008. A pilot study on wound healing using an antibacterial steroidal saponin. Int. J. Biol. Chem. Sci., 2(3): 299-305. DOI: 10.4314/ijbcs.v2i3.39757. Awaad AS, Soliman GA, El-Sayed DF, ElGindi OD, Alqasoumi, SI. 2012. Hepatoprotective activity of Cyperus alternifolius on carbon tetrachloride-induced hepatotoxicity in rats. Pharmaceutical Biology, 50(2): 155-161. DOI:

10.3109/13880209.2011.580351.

Awouafack MD, Mcgaw L, Gottfried S, Eloff JN. 2013. Antimicrobial activity and cytotoxicity of the ethanol extract 
fractions and eight coupounds isolated from Eriosema robustum (Fabaceae). BMC Complementary and Alternative Medecine, 13(1): 289. DOI: https://doi.org/10.1186/1472-6882-13289.

Balouiri M, Sadiki M, ilbnsouda SK. 2016. Methods for in vitro evaluating antimicrobial activity: A review. Journal of Pharmaceutical Analysis, 6: 71-79. DOI:

https://doi.org/10.1016/j.jpha.2015.11.00 5.

Bashige CV, Bakari AS, Numbi IE, Kalonda ME, Okusa NP, Kahumba BJ, Lumbu SJB. 2018. Criblage Phytochimique et activité antimicrobienne de sept fleurs comestibles utilisées en médecine traditionnelle à Lubumbashi (RDC). Journal of Applied Biosciences, 124: 12455-12467.

DOI:

https://dx.doi.org/10.4314/jab.v124i1.7

Bashige-Chiribagula V, Bakari-Amuri S, Mbuyi-Kalonji S, Kahumba-Byanga J, Duez P, Lumbu-Simbi JB. 2017b. Étude ethnobotanique, phytochimique et évaluation de l'activité antiplasmodiale de 13 plantes réputées antipaludéennes dans la commune Kenya (Lubumbashi, RDC). Phytothérapie, 2017 :1-10. DOI : https://doi.org/10.1007/s10298-0171152-x

Bashige-Chiribagula V, Manya-Mboni $\mathrm{H}$, Ntabaza-Ndage V, Numbi IE, BakariAmuri S, Kalonda Mutombo E, Kahumba-Byanga J, Okusa-Ndjolo P, Duez P, Lumbu-Simbi JB. 2017a. Étude ethnobotanique, biologique et chimique de plantes réputées anticariogènes à Lubumbashi-RD Congo. Phytothérapie, 15 : $2-9 . \quad$ DOI : https://doi.org/10.1007/s10298-0151004-5

Carbonnelle B, Denis F, Marmonier A, Pinon G, Vague R 1987. Bactériologie Médicale: Techniques Usuelles. Edition SIMEP: Paris ; 228-282.
Elechi NA, Igboh OT. 2017. Antibacterial activities of the methanol extract and fractions of the leaf of Eriosema psoraleoides (Lam.) G. Don (Leguminosae). International Journal of Pharmaceutical Siences and Research, 8(2): 698-705.

DOI: https://doi.org/10.9734/ajrb/2019/ v4i130061.

Gatsing D, Mbah JA, Garba IH, Tane P, Djemgou P, Nji-Nkah BF. 2006. An Antisalmonellal agent from the leaves of Glossocalyx brevipes Benth (Monimiaceae). Pakistan J Biol Sci, 9: 84-87.

Gleńsk M, Tichaczek-Goska D, ŚrodaPomianek K, Włodarczyk M, Wesolowski CA, Wojnicz D. 2019. Differing antibacterial and antibiofilm properties of Polypodium vulgare $\mathrm{L}$. Rhizome aqueous extract and one of its purified active ingredients-osladin. Journal of Herbal Medicine, 17: 1-6. DOI:

https://doi.org/10.1016/j.hermed.2019.10 0261.

Gupta SK, Sharma A. 2014. Medicinal properties of Zingiber officinale Roscoe A Review. Journal of Pharmacy and Biological Sciences, 9(5): 124-129. DOI: 10.9790/3008-0955124129.

Haniadka R, Saldanha E, Sunita V, Palatty PL, Fayad R, Baliga MS. 2013. A review of the gastroprotective effects of ginger (Zingiber officinale Roscoe). Food Funct., 4: 845-55. DOI: $10.1039 / \mathrm{c} 3$ fo $30337 \mathrm{c}$.

Hoşgor LM, Ermertcan S, Eraç B, Taşli H. 2011. An investigation of the antimicrobial impact of drug combinations against Mycobacterium tuberculosis strains. Turk J Med Sci, 41: 719-724. DOI: 10.3906/sag-1007-934.

Hwang S. 2019. Traditional medicine, modern science, and the upgrading of the local pharmaceutical industry: endogenous development of the phytomedicine 
innovation system in Korea. Innovation and Development 1-19. DOI: https://doi.org/10.1080/2157930X.2019. 1679953

Kaya O, Akçam F, Yaylı G. 2012. Investigation of the in vitro activities of various antibiotics against Brucella melitensis strains. Turk J Med Sci, 42: 145-148. DOI : 10.3906/sag-1009-1129.

Kuete V. 2010. Potential of Cameroonian plants and derived products against microbial infections: A review. Planta Med 76(14): 1479-1491. DOI: $10.1055 / \mathrm{s}-$ 0030-1250027.

Lalthanpuii PB, Zarzokimi, Lalchhandama K. 2018. Imperata cylindrica: a noxious weed of pharmacological potentials. Mizoram Science Congress 2018 (MSC 2018) Atlantis Press. Advances in Engineering Research, 178: 173-177. DOI: $\quad$ https://doi.org/10.2991/msc18.2018.28.

Lalthanpuii PB, Zarzokimi, Lalchhandama K. 2019. Chemical profiling, antibacterial and antiparasitic studies of Imperata cylindrica. Journal of Applied Pharmaceutical Science, 9(12): 117-121. DOI: $10.7324 / J A P S .2019 .91216$.

Longanga O, Vercruysse A, Foriers A. 2000. Contribution to the ethno botanical, phytochemical and pharmacological studies of traditionally used medicinal plants in the treatment of dysentery and diarrhoea in Lomela area, Democratic Republic of Congo (DRC). Journal of Ethnopharmacology, 71: 411-423. DOI: https://doi.org/10.1016/S03788741(00)00167-7.

Madrid L, Seale AC, Kohli-Lynch M, Edmond K M, Lawn JE, Heath PT, Madhi SA, Baker CJ, Bartlett L, Cutland C, Gravett MG, Ip M, Le Doare K, Rubens CE, Saha SK, Sobanjo-ter Meulen A, Vekemans J, Schrag S. 2017. Infant GBS Disease Investigator Group, Infant Group B Streptococcal Disease Incidence and
Serotypes Worldwide: Systematic Review and Meta-analyses. Clinical Infectious Diseases, 65 (S2): S160-S172. DOI: https://doi.org/10.1093/cid/cix656.

Mustapha BA, Kubmarawa D, Shagal MH and Ardo BP. 2016. Preliminary Phytochemical Screening of Medicinal Plants Found in the Vicinity of Quarry Site in Demsa, Adamawa State, Nigeria. American Chemical Science Journal, 11(2): $\quad 1-7 . \quad$ DOI: 10.9734/ACSJ/2016/21519.

Nadimpalli S. 2016. Should the use of complementary and alternative medicine be restricted? Int J Basic Clin Pharmacol, 5: 1691-1694. DOI: http://dx.doi.org/10.18203/23192003.ijbcp20163190.

Plummer S, Weaver MA, Harris JC, Dee P, Hunter J. 2004. Clostridium difficult pilot study: effects of probiotic supplementation on the incidence of $\mathrm{C}$. difficult diarrhea. Microbiol., 2004: 5962.

Raja M, Ravikumar S, Gnanadesigan M, Vijayakumar V. 2010. In vitro antibacterial activity of diterpene and benzoxazole derivatives from Excoecaria agallocha L. Int. J. Biol. Chem. Sci., 4(3): 692-701.

DOI: $10.4314 /$ ijbcs.v4i3.60494.

Schwarz S, Loeffler A, Kadlec K. 2017. Bacterial resistance to antimicrobial agents and its impact on veterinary and human medicine. In Advances in Veterinary Dermatology, Torres SMF, Roudebush P (Eds.). John Wiley \& Sons: Oxford; 95-110. DOI: https://doi.org/10.1002/978111927 8368.ch5.1

Seale AC, Bianchi-Jassir F, Russell NJ, KohliLynch M, Tann CJ, Hall J, Madrid L, Blencowe $\mathrm{H}$, Cousens S, Baker $\mathrm{CJ}$, Bartlett L, Cutland C, Gravett MG, Heath PT, Ip M, Le Doare K, Madhi SA, Rubens CE, Saha SK, Schrag SJ, Sobanjo-ter 
Meulen A, Vekemans J, Lawn JE. 2017. Estimates of the Burden of Group B Streptococcal Disease Worldwide for Pregnant Women, Stillbirths, and Children. Clinical Infectious Diseases, 65(S2): $\quad$ S200-S219 DOI: https://doi.org/10.1093/cid/cix664.

Sima-Obiang C, Ngoua-Meye-Misso RL, Ndong-Atome GR, Privat Ondo J, Obame-Engonga LC, Nsi-Emvo E. 2018. Phytochemical Screening, Phenolic Compounds Content, Antioxidant, Antiinflammatory, and Antimicrobial Properties of Pachylobus balsamifera Guillaum from Gabon. Phytothérapie, 16: S65-S73. DOI: https://doi.org/10.3166/phyto-20180059.

Tatsimo SJN, Tamokou J-D, Tsague VT, Lamshoft M, Sarkar P, Bag PK, Spitelle M. 2017. Antibacterial-guided isolation of constituents from Senna alata leaves with a reference against Multi-DrugResistant Vibrio cholerae and Shigella flexneri. Int. J. Biol. Chem. Sci., 11(1): 46-53.

DOI: http://dx.doi.org/10.4314/ijbcs.v11i1.4

Umair M, Altaf M, Abbasi AM. 2017. An ethnobotanical survey of indigenous medicinal plants in Hafizabad district, Punjab-Pakistan. PLoS ONE, 12(6): e0177912.

DOI: 10.1371/journal.pone.0177912

Varghese A, Gavani U, Abraham S, Parambi DGT, Sathianarayanan and Jose A (2009). Phytochemical screening and antimicrobial investigation of Typha angustifolia LINN. Int. J. Chem. Sci., 7(3): 1905-1910. DOI: 10.4103/02537613.103303 .

WHO. 2003. Manual for the laboratory identification and antimicrobial susceptibility testing of bacterial pathogens of public health importance in the developing world: Haemophilus influenzae, Neisseria meningitidis, Streptococcus pneumoniae, Neisseria gonorrhoea, Salmonella serotype Typhi, Shigella, and Vibrio cholerae / Principal authors: Perilla MJ [Expert]. World Health Organization: Genève, Suisse. https://apps.who.int/iris/handle/10665/68 554.

William YN, Gilbert A, Shah AJ, Marius M, Tchoumba TLM, Matah MMV, Adjouzem CF, Teboukeu BG, Awouafack MD, Taous K, Kamanyi A. 2019. Extracts from the trunk bark of Distemonanthus benthamianus Baillon. (Caesalpiniaceae) developed antidiarrhoeal activities in rats and mice. Orient Pharm Exp Med, 19: 421-433. DOI: https://doi.org/10.1007/s13596019-00385-x.

Zhang LL, Zhang LF, Hu QP, Hao DL, Xu J.G. 2017. Chemical composition, antibacterial activity of Cyperus rotundus rhizomes essential oil against Staphylococcus aureus via membrane disruption and apoptosis pathway. Food Control, 80: 290-296. DOI: https://doi.org/10.1016/j.foodcont.2017.0 5.016. 